\title{
Correction
}

\section{Correction to: Numerical study of non-linear thermal radiative heat transfer in a non-Darcy chemically reactive Casson fluid flow}

\author{
G. Sarojamma ${ }^{1} \cdot$ K. Sreelakshmi ${ }^{1} \cdot$ I. L. Animasaun ${ }^{2}$
}

(c) Springer Nature Switzerland AG 2019

\section{Correction to: SN Applied Sciences (2019) 1:1136}

https://doi.org/10.1007/s42452-019-1159-z

The partial derivative DOW is missing in Eqs. (3-5) in the original publication. The corrected equations are shown below.

$$
\begin{gathered}
\frac{1}{\varepsilon^{2}}\left(u \frac{\partial u}{\partial x}+v \frac{\partial u}{\partial y}\right)=\frac{v}{\varepsilon}\left(1+\frac{1}{\beta}\right) \frac{\partial^{2} u}{\partial y^{2}}+\frac{\sigma}{\rho}\left(E_{0} B_{0}-B_{0}^{2} u\right) \\
-\frac{v}{k_{1}} u-\frac{C_{b}}{\sqrt{k_{1}}} u^{2}+g \beta_{T}\left(T-T_{\infty}\right)+g \beta_{C}\left(C-C_{\infty}\right) \\
u \frac{\partial T}{\partial x}+v \frac{\partial T}{\partial y}=\frac{k}{\rho C_{p}} \frac{\partial^{2} T}{\partial y^{2}}+\frac{16 \sigma^{*}}{3 k^{*} \rho C_{p}} \frac{\partial}{\partial y}\left(T^{3} \frac{\partial T}{\partial y}\right) \\
+\frac{\mu}{\rho C_{p}}\left(1+\frac{1}{\beta}\right)\left(\frac{\partial u}{\partial y}\right)^{2}+\frac{\sigma}{\rho C_{p}}\left(u B_{0}-E_{0}\right)^{2}
\end{gathered}
$$

$$
u \frac{\partial C}{\partial x}+v \frac{\partial C}{\partial y}=D \frac{\partial^{2} C}{\partial y^{2}}+\frac{D k_{T}}{T_{m}} \frac{\partial^{2} T}{\partial y^{2}}-k_{0}\left(C-C_{\infty}\right)
$$

Publisher's Note Springer Nature remains neutral with regard to jurisdictional claims in published maps and institutional affiliations.

The original article can be found online at https://doi.org/10.1007/s42452-019-1159-z.

G. Sarojamma, gsarojamma@gmail.com; K. Sreelakshmi, katasreelakshmi@gmail.com; I. L. Animasaun, ilanimasaun@futa.edu.ng | 'Department of Applied Mathematics, Sri Padmavati Mahila Visvavidyalayam, Tirupati 517502, India. ${ }^{2}$ Department of Mathematical Sciences, Federal University of Technology, Akure, Nigeria. 